\title{
TEMPERATURA Y CONCENTRACIÓN DE FLUORURO DE SODIO EN GELES FLUORADOS
}

\author{
Temperature and concentration of sodium fluoride in fluorid gels
}

\author{
Vianne Diana Huamán Baca ${ }^{1}$, Alejandro Pletickosich Picón ${ }^{1,-[}$, Teófila Baca Carbajal². \\ ${ }^{1}$ Universidad Andina del Cusco, Cusco, Perú. \\ ${ }^{2}$ Escuela Profesional de Química, Universidad Nacional de San Antonio Abad del Cusco, Cusco, Perú. \\ Ðapletickosich@uandina.edu.pe
}

\begin{abstract}
Resumen
El objetivo de esta investigación fue determinar la influencia de la temperatura en la concentración de fluoruro de sodio; la muestra estuvo conformada por 60 geles fluorados de un mismo lote y la validez de dos marcas: 'Fluofar®' en sus dos presentaciones (pH neutro y acidulado) y el 'Fluorgel Maquira ${ }^{\circledR}$ ' en sus dos presentaciones ( $\mathrm{pH}$ neutro y acidulado). De cada gel fluorado se obtuvieron cuatro muestras: para comprobar el $\mathrm{pH}$, para determinar la concentración de fluoruro de sodio a temperatura ambiente, para fijar la concentración de fluoruro de sodio después de haber sido sometido a una temperatura de $8{ }^{\circ} \mathrm{C}$ durante tres meses, y para, determinar la concentración de fluoruro de sodio después de haber sido sometido a una temperatura de $20^{\circ} \mathrm{C}$ durante tres meses.

La determinación de concentración de fluoruro de sodio, se realizó mediante la técnica de destilación y titulación. Por los resultados obtenidos se concluyó: que el incremento de la temperatura influye en la disminución de la concentración de fluoruro de sodio — siendo estadísticamente significativa en los geles fluorados con pH ácido (geles acidulados) y, en los geles fluorados con pH neutro mantiene su concentración-, aún se incrementó la temperatura. La concentración de fluoruro de sodio, indicado por el fabricante, disminuyó significativamente al comparar con la concentración de fluoruro de sodio encontrado en el laboratorio.

Palabras claves; Geles fluorados, concentración de fluoruro de sodio, temperatura, $\mathrm{pH}$
\end{abstract}

\section{Abstract}

The objective of this investigation was to determine the influence of temperature on the concentration of sodium fluoride; the sample consisted of 60 fluorinated gels from the same batch and validity of two brands: Fluofar ${ }^{\circledR}$ in its two presentations (neutral and acidulated pH) and Fluorgel Maquira ${ }^{\circledR}$ and its two presentations (neutral $\mathrm{pH}$ and acidulated). From each fluorinated gel four samples were obtained: to determine the $\mathrm{pH}$, to determine the concentration of sodium fluoride at room temperature, to determine the concentration of sodium fluoride after having been subjected to temperature of $8{ }^{\circ} \mathrm{C}$ for three months, and to determine the concentration of sodium fluoride after having been subjected to temperature of $20{ }^{\circ} \mathrm{C}$ for three months.

The determination of concentration of Sodium Fluoride was carried out by means of the distillation and titration. From the results obtained, it was concluded that the increase in temperature influences the decrease in the concentration of sodium fluoride -being statistically significant in fluorinated gels with acidic $\mathrm{pH}$ (acidified gels) and, in fluorinated gels with neutral $\mathrm{pH}$, it maintains its concentration-, the temperature was still increased. The concentration of sodium fluoride, indicated by the manufacturer, decreased significantly when compared to the concentration of sodium fluoride found in the laboratory.

Keywords: Fluorinated gels, sodium fluoride concentration, temperature, $\mathrm{pH}$.

Citar como: Huaman, V., Pletickosich, A., Baca, T. (2018). Temperatura y concentración de fluoruro de sodio en geles fluorados. Rev Yachay, 7(1), 294-301.

Recibido: 16-09-2018; Aceptado 07-12-2018

\section{Introducción}

En el Perú, la caries afecta al 95\% de habitantes, siendo los niños en etapa escolar comprendida entre los 6 y 12 años de edad, quienes se encuentran en proceso de recambio dentario, afectándolos en un $84 \%$. Estas cifras colocan al Perú entre los países latinoamericanos con mayores niveles de esta enfermedad (Reyes Ruiz, 2012). Ante esta problemática, el flúor constituye un excelente complemento para la

Rev Yachay volumen (7) Numero (1), enero-diciembre 2018 
prevención de caries dental gracias a que puede inhibir la formación de ácidos por las bacterias, aumentando de esta manera, el pH salival y transforma los cristales de hidroxiapatita por fluorapatita. De esta manera, se evita el proceso de desmineralización del esmalte dando como resultado una estructura más resistente a la caries dental; siendo esto, beneficioso en pacientes con caries (Boj, JR.; Catalá , M.; García-Ballesta , C.; Mendoza, A., 2005).

El objetivo de la investigación fue determinar la influencia de la temperatura en la concentración de fluoruro de sodio en geles fluorados expendidos en las boticas dentales del distrito de Cusco.

La presente investigación se justifica por la relevancia social, puesto que los resultados de la investigación no solo benefician a la comunidad científica, sino también a la población en general, habida cuenta que este producto es de uso común y necesario para la prevención, manipulación y uso de los productos investigados. La hipótesis que se planteó, fue determinar si el incremento de la temperatura influye en la disminución de la concentración de fluoruro de sodio en geles fluorados expendidos en las boticas dentales del distrito de Cusco.

La contribución de este estudio radica en alertar sobre algunas implicancias que puedan tener los resultados de esta investigación, ya que, en principio: la concentración reportada de fluoruro de sodio en el rotulado del producto, según las marcas estudiadas con los resultados encontrados en laboratorio, tienen una variación significativa en ambas presentaciones (acidulada y neutro); lo que amerita la supervisión de los entes reguladores.

\section{Materiales y métodos}

El diseño de la investigación es cuasi experimental, su unidad de estudio estuvo determinada por los geles fluorados, con un muestreo de tipo no probabilístico por conveniencia, la misma estuvo conformada por 60 geles fluorados de dos diferentes marcas: 'FLUOFAR' (acidulado y neutro) y 'FLUORGEL MAQUIRA' (acidulado y neutro); del mismo lote y validez con concentración de 1,23 y $2 \%$ de fluoruro de sodio, adquiridas en 5 boticas dentales del distrito de Cusco. Luego, se procedió al traslado físico de las muestras del lugar de adquisición por vía terrestre hasta el laboratorio, donde se efectuaron las condiciones de conservación y de protección mínima contra los riesgos ambientales y físicos de la muestra. Se realizó, de tal manera que se impidió su ruptura, derrame, alteración, deterioro y la exposición a la luz directa por medio de un recipiente conservador hermético y, el tiempo máximo de transporte fue antes de las 24 horas, desde el lugar de adquisición de los geles fluorados hasta el laboratorio, cumpliendo las recomendaciones de la Directiva Sanitaria MINSA/DIGESA.

\section{Procedimiento:}

$\checkmark$ Se extrajeron 4 muestras de gel, cada una de ellas de 15 gramos y se colocaron en vasos de precipitado. Se ha determinado el $\mathrm{pH}$ y la concentración de fluoruro de sodio a temperatura ambiente $12{ }^{\circ} \mathrm{C}$, inmediata a su adquisición. Luego, se sometió a temperaturas de $8{ }^{\circ} \mathrm{C}$ y $20^{\circ} \mathrm{C}$ en equipos de refrigeración previamente calibrados, por el lapso de tres meses (enero, febrero y marzo de 2017 ); posteriormente, se determinó la concentración de fluoruro de sodio.

El protocolo del procedimiento experimental de laboratorio que se tuvo a considerar fue el del laboratorio MC QUIMICALAB.

- Se colocó en un vaso de precipitado y se agregó $10 \mathrm{ml}$ de agua destilada.

- Se agitó hasta lograr una dilución homogénea.

- Se colocó el pHmetro automático para su respetiva lectura, el valor dado fue consignado en la ficha de recolección de datos.

Preparación de la muestra se pesa $10,7 \mathrm{~g}$ de gel fluorado al que se le agrega agua destilada, agitándolo hasta lograr una dilución homogénea. Aforar a 100ml en una fiola. Esta dilución se trasvasa a un frasco de plástico, el cual se aseguró con una tapa hermética y se rotuló.

\section{Equipos y reactivos:}

a. Equipo de destilación.

b. Ácido sulfúrico $\left(\mathrm{H}_{2} \mathrm{SO}_{4}\right)$.

c. Sulfato de plata $\left(\mathrm{Ag}_{3} \mathrm{SO}_{4}\right)$.

\section{Procedimiento:}

$\checkmark \quad$ En un balón de destilación se colocan $200 \mathrm{ml}$ de ácido sulfúrico concentrado y $400 \mathrm{ml}$ de agua destilada y cristales de sulfato de plata.

$\checkmark \quad$ Luego, se agrega 25 perlas de vidrio y se destila a $180{ }^{\circ} \mathrm{C}$ para eliminar contaminantes de flúor.

$\checkmark \quad$ Se descarta el destilado y se agregan $100 \mathrm{ml}$ de la muestra (gel diluido en agua destilada).

Rev Yachay volumen (7) Numero (1), enero-diciembre 2018 
Se repite la destilación hasta que todo el fluoruro haya destilado.

La muestra destilada se llena en una probeta de plástico y se rotula.

\section{Cuantificación del fluoruro de sodio}

La cuantificación del fluoruro de sodio en la muestra destilada se realiza de la siguiente forma:

Titulación en blanco (patrón).

- Titulación de la muestra

Equipos y reactivos:

$\checkmark$ Equipo de titulación

$\checkmark$ Bureta

$\checkmark$ Pipetas

$\checkmark$ Erlenmeyer

$\checkmark \mathrm{CaCl}_{2}=0,09 \mathrm{M}$

$\checkmark$ EDTA $=0,02 \mathrm{~N}$ (titulante)

$\checkmark$ Negro de eriocromo T (indicador)

$\checkmark$ Agua destilada

$\checkmark$ pH 10 amortiguador

- Titulación en blanco (patrón):

- Se mide un mililitro de $\mathrm{CaCl}_{2}$ reactivo, se agrega agua destilada y procede a titular con EDTA.

- $\quad$ Se lee el volumen gastado $=\mathrm{V}_{1}$.

1. Titulación de la muestra:

Procedimiento:

De la muestra destilada medir $10 \mathrm{ml}$, se coloca a un vaso Erlenmeyer, se agrega $1 \mathrm{ml} \mathrm{de} \mathrm{CaCl}_{2}, 10 \mathrm{ml}$ de agua destilada, 10 gotas de pH 10 amortiguador y 5 gotas de indicador, agitar hasta que la mezcla torne a un color púrpura.

Se inicia con el método de titulación gota a gota, hasta observar cambio de color: de púrpura a azul y leer el volumen gastado $=\mathrm{V}_{2}$.

\section{Cálculo para determinar la concentración de fluoruro de sodio}

- $\quad \mathrm{V}_{1}-\mathrm{V}_{2}=\mathrm{V}_{3}$

Este volumen es el que reaccionó con el fluoruro de sodio.

- $\quad$ Por definición se sabe que:

Donde:

$\mathrm{N}=$ Normalidad

$\mathrm{V}=$ Volumen

$\mathrm{VN}=\mathrm{n} .^{\circ}$ de miliequivalentes

- $\quad$ En este caso: $\mathrm{V}_{3} \mathrm{~N}=\mathrm{n} .^{\circ}$ de miliequivalentes de $\mathrm{NaF}$.

- Para convertir a mg se tiene:

n. ${ }^{\circ}$ de miliequivalentes de $\mathrm{NaF}$ x $42=$ mg.NaF.

- Para destilar se ha tomado un peso de la muestra $=P_{0}$

Para convertir a $\%$ se tiene:

$$
\frac{\mathrm{N} .^{\circ} \text { de gr de NaF }}{\text { P0 }} \times 100=\%
$$

Paso 9: Los resultados de las concentraciones obtenidas mediante el cálculo de las ecuaciones se registró en la ficha de recolección de datos, para el análisis estadístico.

Todo material, reutilizable y no reutilizable contaminado, fue lavado y esterilizado, el almacenaje de los desechos para su eliminación fue en bolsas de plástico de acuerdo a la clase de residuos. 


\section{Validez y confiabilidad de los instrumentos}

El instrumento de recolección de datos fue validado por tres expertos:

01 Químico

01 Cirujano dentista

01 Metodólogo

Para tal efecto, se acompaña la ficha de recolección de datos y las fichas de validación para expertos. (Ver Anexo 6).

\section{Plan de análisis de datos}

Los datos obtenidos fueron procesados siguiendo un patrón de tabulación automatizado con el soporte del paquete estadístico SPSS (Programa Estadístico para Ciencias Sociales) versión 22.0. Los resultados se reportaron en cuadros estadísticos de entrada doble de acuerdo a los objeticos señalados.

Para determinar la influencia de la temperatura sobre la concentración de fluoruro de sodio en las muestras de geles, se aplicó la técnica estadística de la t de Student, tomando como parámetro el valor rotulado; para comparar el pH entre los diferentes geles fluorados, se aplicó la prueba $\mathrm{F}$ del análisis de varianza.

\section{Resultados}

Figura 1. Concentración de fluoruro de sodio (\%) indicado por el fabricante y concentración de fluoruro de sodio (\%) determinados en laboratorio en geles fluorados.

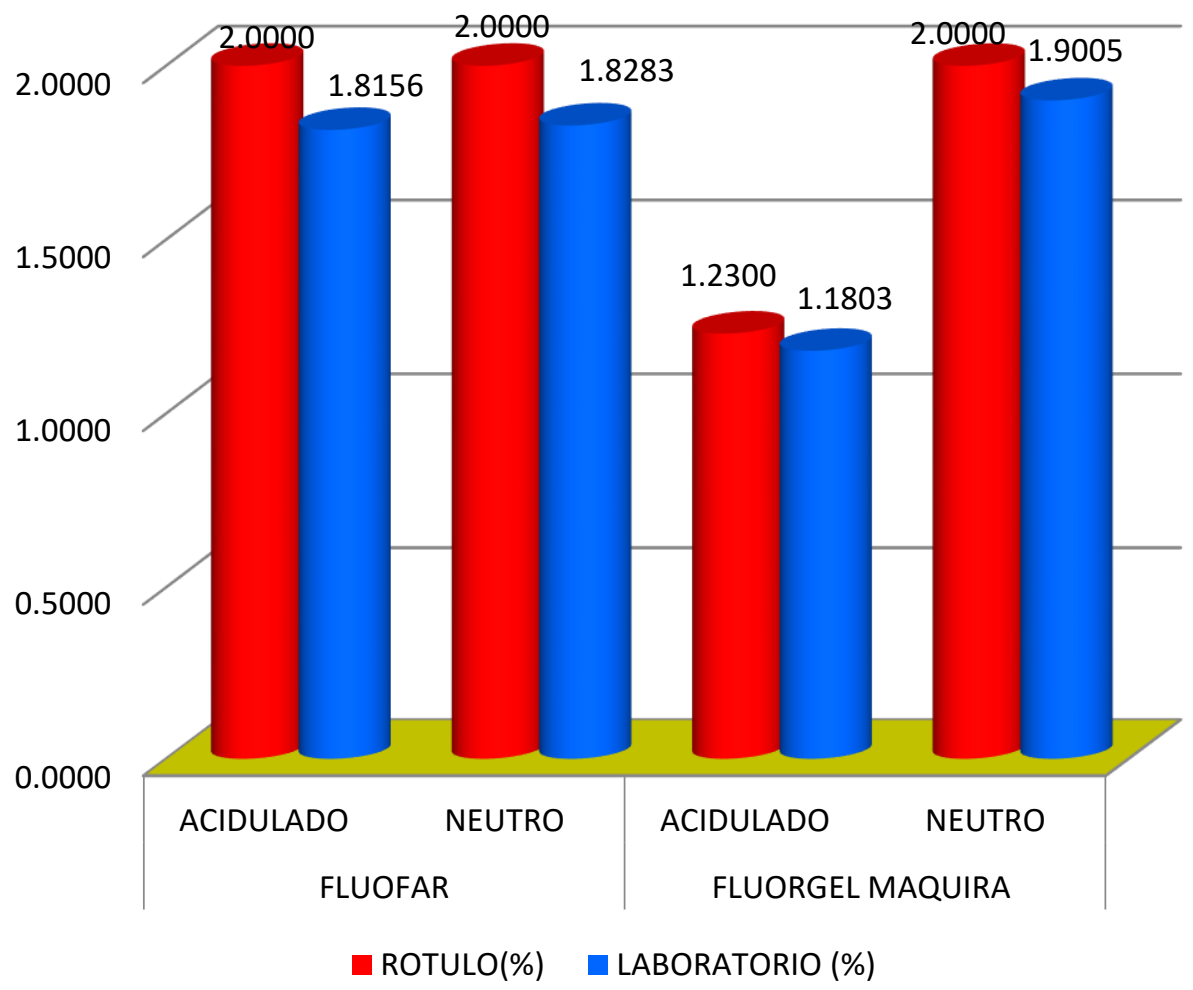

Fuente: Ficha de recolección datos

La figura1, presenta la concentración de fluoruro de sodio indicado por el fabricante y concentración promedio de fluoruro de sodio determinados a nivel de laboratorio, llegando a observar la marca Fluofar ${ }^{\circledR}$ (rotulado) en muestra acidulada y neutra un $2 \%$ de

Rev Yachay volumen (7) Numero (1), enero-diciembre 2018 
concentración de fluoruro de sodio para ambos casos, pero, para los registros en el laboratorio se tiene: para muestra acidulada 1,8156\% $\mathrm{y}$, para neutra $1,8283 \%$; existiendo una diferencia significativa.

Por otro lado, la marca FlúorGel Maquira®: para rotulado 1,2300\% de concentración para muestra acidulada y de 2\% de concentración para la muestra neutra y, según el registro a nivel de laboratorio, en la muestra acidulada se encontró 1,1803\% y para la muestra neutra $1,9005 \%$ de concentración de la mencionada sustancia.

En ambos tipos de muestras de gel existe diferencia significativa en la concentración de fluoruro de sodio.

Figura 2: Concentración de fluoruro de sodio (\%) según las dos condiciones de temperatura: $8{ }^{\circ} \mathrm{C}$ y $20{ }^{\circ} \mathrm{C}$.

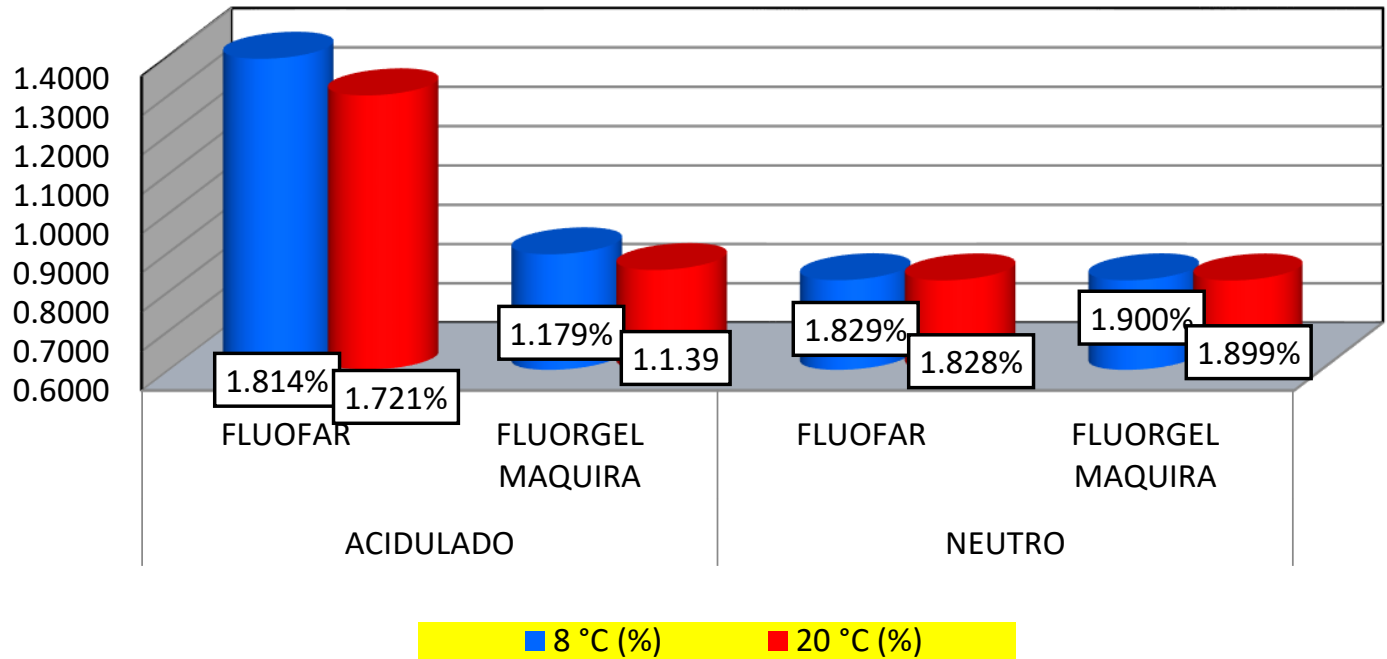

Fuente: Ficha de recolección datos

Los resultados de la figura 02 , presentan la concentración de fluoruro de sodio según las condiciones de temperatura $\left(8^{\circ} \mathrm{C}\right.$ y $20{ }^{\circ} \mathrm{C}$, en el lapso de 3 meses) para las muestras aciduladas y neutras.

Teniendo para las muestras aciduladas a 8 grados centígrados en la marca Fluofar ${ }^{\circledR}$ 1,814\% de concentración de fluoruro de sodio y para la marca FlúorGel Maquira ${ }^{\circledR}, 1,179 \%$. En tanto, para una temperatura de $20{ }^{\circ} \mathrm{C}$ en Fluofar ${ }^{\circledR}$, se obtiene un $1,721 \%$ y para la marca FlúorGel Maquira® 1,139\%.

Para las muestras neutras a temperatura de $8{ }^{\circ} \mathrm{C}$, para la marca Fluofar ${ }^{\circledR} 1,829 \%$ y para la marca FlúorGel Maquira ${ }^{\circledR} 1,900 \%$, de concentración en la sustancia estudiada; para las muestras neutras a $20{ }^{\circ} \mathrm{C}$, en la marca Fluofar ${ }^{\circledR} 1,828 \%$, y para la marca FlúorGel Maquira® 1,899\% de fluoruro de sodio.

Según los resultados presentados la concentración de fluoruro de sodio en muestras aciduladas para las temperaturas de $8{ }^{\circ} \mathrm{C}$ y $20{ }^{\circ} \mathrm{C}$ tienen diferencias significativas; en el caso de las muestras neutras, las diferencias son mínimas o poco significativas.

\section{Discusión}

En la presente investigación se evidencia que la concentración de fluoruro, declarado en el rótulo de los geles de estudio, difieren con los resultados evaluados en el laboratorio a temperatura ambiente.

Estos resultados son similares a los reportados por Ricomini, F., Tenuta L., Fernandes S., Calvo A., Cury (2012), quienes demuestran que las concentraciones de flúor en las muestras analizadas en pastas de dientes brasileñas más vendidas en sus diferentes regiones

Rev Yachay volumen (7) Numero (1), enero-diciembre 2018 
estuvieron muy cerca a la cantidad adecuada de 1450 ppm de flúor entre 1 035,5 $\pm 61,5$ a $1221,8 \pm 35,2$; que indica en el empaquetado del fabricante.

Hernández G., De la Fente H., Ledesma M., (2005), donde sus resultados muestran que la concentración real de flúor (F) en pastas de dientes que se venden en el mercado mexicano, tienen una amplia variación en concentraciones de este producto, no presentan en el empaque el contenido real del mismo $(\mathrm{F})$.

Hashizume LN, Mathias TC, Cibils DM, Maltz M., (2003), las concentraciones de fluoruro de los dentífricos presentaron similares (no iguales) de fluoruro total en muestras recientes (frescas), que son conformes a la legislación japonesa (contenido: no menor de 1000 ppm de flúor).

Asimismo en el Perú, para Salvatierra García (2014), la concentración de fluoruro encontrado en los dentífricos cosméticos bucales fluorados para niños que se comercializan en el distrito de Trujillo, varían significativamente, hallándose concentraciones muy bajas a lo declarado por el fabricante y con la norma técnica del MINSA.

En lo que se refiere a la concentración de flúor a temperatura, la presente investigación obtuvo:

La concentración de fluoruro de sodio sometido a distintas temperaturas $\left(8{ }^{\circ} \mathrm{C}, 12\right.$ y $\left.20{ }^{\circ} \mathrm{C}\right)$ en geles fluorados expendidos en tiendas dentales del distrito de Cusco, disminuye significativamente, tanto en muestras aciduladas como en pequeñas cantidades en muestras neutras.

\section{Resultados que corroboran:}

Atúncar (2002), presenta que la concentración de flúor determinada mediante un electrodo de ion específico marca Orión modelo EA 920, en muestras sometidas a temperatura ambiente, refrigeración y alza térmica, mostraron que los dentífricos con fluoruro de sodio disminuyeron del 3,3\% de la concentración establecida por el fabricante; mientras que los dentífricos con monofluoruro fosfato de sodio, presentaron una disminución que variaba entre 15,5 y $20 \%$ de la concentración establecida por el fabricante. De la misma manera, luego de 200 días de estar almacenadas bajo las condiciones indicadas observaron que los dos grupos presentaban disminución en sus concentraciones, siendo más notoria en las muestras sometidas bajo alza térmica, variando en un 15,5\% en las muestras que contenían monofluoruro fosfato de sodio en relación a las muestras iniciales; y, en un 2,28\% en los dentífricos con fluoruro de sodio, con relación a sus concentraciones iniciales.

Ayala Colana (2016), concluye que la concentración de fluoruro en pastas dentales frente a la variación de la temperatura ambiental y el tiempo de almacenamiento en los distritos de Yanacancha y Constitución en la Región Pasco, de $-1{ }^{\circ} \mathrm{C}$ a $11^{\circ} \mathrm{C}$; a los 3 y 9 meses de almacenamiento, la concentración de flúor fue: a los 3 meses en la muestra 1: 1367 ppm, muestra 2: 1388 ppm y en la muestra 3: 1372 ppm; a los 9 meses en la muestra 1: $1236 \mathrm{ppm}$, muestra 2: $1259 \mathrm{ppm}$ y en la muestra 3: $1269 \mathrm{ppm}$. A una temperatura ambiental de 25 ${ }^{\circ} \mathrm{C}$ a $33{ }^{\circ} \mathrm{C}$, a los 3 y 9 meses de almacenamiento, la concentración de flúor fue: a los 3 meses en la muestra 1: $1351 \mathrm{ppm}$, muestra 2: 1 384 ppm y, en la muestra 3: 1355 ppm; a los 9 meses en la muestra 1: 1198 ppm, muestra 2: 1252 ppm y en la muestra 3: 1205 ppm. Se tiene que la variación de la temperatura ambiental y el tiempo de almacenamiento influyen en la disminución de la concentración de flúor, siendo estadísticamente significativa la disminución en las muestras almacenadas a temperatura ambiental de entre los $25^{\circ} \mathrm{C}$ a 33 ${ }^{\circ} \mathrm{C}$.

\section{Conclusiones}

Las concentraciones de fluoruro de sodio, según las dos condiciones de temperatura, en geles fluorados expendidos con pH ácido (geles acidulados) disminuyó; en tanto, para los geles fluorados con $\mathrm{pH}$ neutro varían su concentración de manera menos significativa para un incremento de la temperatura.

Se determinó que la concentración de fluoruro de sodio, indicado por el fabricante, disminuyó al comparar con la concentración de fluoruro de sodio encontrado en el laboratorio en geles fluorados expendidos en tiendas dentales del distrito de Cusco.

Rev Yachay volumen (7) Numero (1), enero-diciembre 2018 


\section{Referencias bibliográficas}

Atúncar Guzmán, M. (2002). Concentración de fluoruros contenidos en los dentífricos en función a la temperatura. Lima.

Nolasco, A. (2000). Control de calidad de colutorios bucales fluorados. Lima.

Robinson, C. (2009). Fluoride and the caries lesion: interactions and mechanism of action. Eur Arch Paediatr Dent.

American Dietetic Association. (2000). The impact of fluoride on health. J Am Diet Assoc.

Aparecido , C., Oliveira, M., Martins, C., \& Tenuta, L. (2010). Floururo disponible en los dentríficos usados por los niños brasileños.

Armas, S. (2016). Fluoruro de sodio: usos y efectos secundarios. Obtenido de ONSALUD: https://www.onsalus.com/fluoruro-de-sodio-usos-yefectos-secundarios-18148.html

Atkins, P. (1999). Fisicoquímica. Barcelona: Omega.

Atúncar, M. (2002). Concentracion de fluoruros contenidos en los dentrificos en funcion a la temperatura. Universidad Nacional Mayor de San Marcos, Estomatología. Lima: UNMSM. Recuperado el 4 de noviembre de 2018, de http://cybertesis.unmsm.edu.pe/bitstream/handle/cybertes is/2774/Atuncar_gm.pdf?sequence $=1 \&$ isAllowed $=\mathrm{y}$

Ayala Colana, G. (2016). Concentración de fluoruro en pastas dentales frente a la variación de temperatura ambiental y el tiempo de almacenamiento de los distritos de Yanacancha y Constitución. Pasco-Perú 2014. . Tesis de grado, Universidad Privada Norbert Wiener, Facultad de Ciencias de la Salud Escuela Académico profesional de Odontología, Lima. Recuperado el 4 de noviembre de 2018,

de http://repositorio.upch.edu.pe/bitstream/handle/upch/369 5/Evaluacion_AngelesRomero_Diego.pdf?sequence=1\&i sAllowed $=\mathrm{y}$

Barrancos Moonet, J., \& Barrancos, P. (2006). Operatoria Dental Integración Clínica. Buenos Aires.

Boj, JR.; Catalá , M.; García-Ballesta , C.; Mendoza, A. (2005). Odontopediatría (2. ${ }^{\circ}$ ed.). Barcelona, España: Ed.Masson.

California Dental Association. (2012). Prevenir la caries dental con fluoruro.

Canahuire Montufar, A. E., Endara Mamani, F., \& Morante Ríos, E. (2015). ¿Como hacer la tesis universitaria? Cusco: Colorgraf S.R.L.

CC Marinho, V., \& PT Higgins, J. (2002). https://www.epistemonikos.org. Obtenido de

Rev Yachay volumen (7) Numero (1), enero-diciembre 2018 https://www.epistemonikos.org/es/documents/2ec306db4 abe3d1cf2b38ee2a6c1c37712a5b8e8

Cortes Ibarra, M., \& Garibay Sánchez , R. (2015). Temperatura.

Enciclopediasalud. (02 de Febrero de 2016). Enciclopediasalud. Obtenido de http://www.enciclopediasalud.com/definiciones/fluoruro

FISICALAB. (10 de Mayo de 2017). https://www.fisicalab.com. Obtenido https://www.fisicalab.com/apartado/temperatura\#conteni dos

García , J. (2009). Absorción sistémica de flúor en niños secundaria al cepillado con dentífrico fluorado. Rev Esp Salud Pública.

García Sobrino, M. (19 de Setiembre de 2011). El flúor, pros y contras. Obtenido de http://www.gacetadental.com/2011/09/el-flor-pros-ycontras-25531/

Gómez Santos, G., Gómez Santos, D., \& Martín Delgado, M. (2002). Flúor y fluorisis dental. Santa Cruz de Tenerife: Dirección General de Salud Pública. Servicio Canario de la Salud.

Gonzáles , A. (1999). Bases para el uso racional del flúor en la prevención y tratamiento de caries en pediatría. Revista Pediátrica de Atención Primaria.

González Toro, C. (2011). Monitoreo de la calidad del agua. Lima. Hashizume LN, Mathias TC, Cibils DM, Maltz M. (20 de febrero de 2003). Effect of the widespread use of fluorides on the occurrence of hidden caries in children. Int $J$ Paediatr Dent., 23(1), 72-76. doi:10.1111/j.1365263X.2012.01231.x

Heredia , C. (1999). Odontología preventiva en el niño y en el adolescente. . Lima: Editorial universidad Cayetano Heredia.

Hernández G., De la Fente H., Ledesma M. (2005). Concentración de fluor en las pastas dentales que se venden en México. (UNAM, Ed.) Boletín Médico Hospital Infantil México, 62(1), 19-24. Recuperado el 4 de noviembre de 2018, de http://www.scielo.org.mx/scielo.php?pid=S1665$11462005000100004 \&$ script $=$ sci_abstract

Hernandez, R., \& Fernández, C. B. (2014). Metodologìa de la investigación (Quinta ed.). México: Mc Graw Hill.

Instituto Asturiano De Odontologia S.I. (2005). Manual tecnico superior de higiene bucodental. Obtenido de Http://Books.Google.Com.Pe

Iruretagoyena, M. (2009). El fluoruro y los diferentes vehículos para prevenir la caries dental. Obtenido de http://www.sdpt.net/CAR/fluoruros.htm\#Geles 
Jenkins, G. (1983). Fisiología y bioquímica bucal . México: 4. ${ }^{\circ}$ Edición Editorial LIMUSA .

López, M. (1997). "Manual de Odontopediatría” . Editorial Mc Graw Hill

Mamani Quisbert, C., \& Zeballos López , L. (2012). Cariostaticos. Revista de Actualización Clínica Investiga.

Mc Donald, K. (1994). Odontología preventiva en acción. México: Editorial Panamericana.

Medina Solís , C., Villalobos Robledo , J., Márquez Corona , M., López Portillo Núñez , C., \& Casanova Rosado , A. (2009). Desigualdades socioeconómicas en la utilización de servicios de salud bucal: estudios en escolares mexicanos de 6 a 12 años de edad. .

Miñana, V. (2011). Promoción de la salud bucodental . Pediatría Atención Primaria.

Municipalidad Provincial del Cusco. (2015). Plan urbano del distrito de Cusco. Cusco.

Murray, R., Granner, D., \& Mayes, P. (1999). Harper bioquímica ilustrada.

Ogaard , B., Seppa , L., \& Rolla , G. (1994). Professional topical Senamhi. fluoride aplications clinical efficacy and mechanism of action. Adv Dent Res .

Oliveby , A., Twetman , S., \& Ekstrand , J. (1990). fluoride Steimberg, S. (2002). A paradigm shift in the treatment of caries. concentration in whole saliva in children living in a high- Thylstrup , A., \& Fejerskov , O. (1998). Caries. . Barcelona: and a low-fluoride area. . Caries Res.

Organización Mundial de la Salud. (1994). Los fluoruros y la salud Universidad Católica Andrés Bello . (2013). Estilación. bucodental. Ginebra.

PCE Ibérica S.L. (30 de Setiembre de 2014). http://www.pce- White, D. iberica.es/. Obtenido de http://www.pce-iberica.es/

Peña , M. (2007). Temperatura . El Salvador.

Reyes Ruiz, Y. (2012). Diferencias en la concentración de iones Winston, A., \& Bjaskar, S. (1999). Prevención de la caries en el flúor en saliva posterior a la aplicación con barnices de fluoruro de sodio Al 2,26\% F, y fluoruro de silano al Editorial Doyma.

$0,1 \% F$. CONCYTEC, Escuela de Estomatología de la USMDP . Lima: ALICIA (Acceso libre a información científica para la innovación). Recuperado el 3 de noviembre de 2018, de http://www.repositorioacademico.usmp.edu.pe/handle/us $\mathrm{mp} / 728$

F., Tenuta L., Fernandes S., Calvo A., Cury A. (junio de dientes brasileños más vendidos comprados en diferentes regiones. Braz Dent, 23(1), 45-48. Recuperado el 4 de noviembre de 2018, de http://dspace.unitru.edu.pe/bitstream/handle/UNITRU/61 2/SalvatierraGarcia_S.pdf?sequence $=1$ 2013”. Universidad Nacional de Trujillo, Estomatología. Trujillo: UNATRU. Recuperado el 4 de noviembre de 2018, de info:eu-repo/semantics/openAccess www.senamhi.gob.pe/main_down.php?ub=mmt\&id=cap Determinación del grado alcohólico del vino. Guayana .

. (1994). Mode of action of fluoride application of new techniques and methods to the examination of The mechanism of action of topical fluoride. . siglo XXI. Jada. 